\title{
Planckian physics comes into play at Planckian distance from horizon
}

\author{
Pei-Ming Ho, ${ }^{a, b}$ Hikaru Kawai ${ }^{a, b}$ and Yuki Yokokura ${ }^{c}$ \\ ${ }^{a}$ Department of Physics and Center for Theoretical Physics, National Taiwan University, \\ No. 1 Roosevelt Road, Taipei 106, Taiwan, R.O.C. \\ ${ }^{b}$ Physics Division, National Center for Theoretical Sciences, \\ No. 1 Roosevelt Road, Taipei 106, Taiwan, R.O.C. \\ ${ }^{c} i T H E M S$ Program, RIKEN, \\ 2-1 Hirosawa, Wako, Saitama 351-0198, Japan \\ E-mail: pmho@phys.ntu.edu.tw, hikarukawai@phys.ntu.edu.tw, \\ yuki.yokokura@riken.jp
}

ABSTRACT: In the background of a gravitational collapse, we compute the transition amplitudes for the creation of particles for distant observers due to higher-derivative interactions in addition to Hawking radiation. The amplitudes grow exponentially with time and become of order 1 when the collapsing matter is about a Planck length outside the horizon. As a result, the effective theory breaks down at the scrambling time, invalidating its prediction of Hawking radiation. Planckian physics comes into play to decide the fate of black-hole evaporation.

KeYwords: Black Holes, Effective Field Theories

ArXiv EPRINT: 2111.01967 


\section{Contents}

1 Introduction 1

2 Review of scalar field in Schwarzschild background 3

3 Trans-Planckian problem $\quad 5$

$\begin{array}{lll}4 & \text { Energy conservation and uncertainty relation } & 7\end{array}$

5 Particle creation by higher-derivative interactions 11

$\begin{array}{lll}6 & \text { Infalling particle radiation } & 15\end{array}$

$\begin{array}{lll}7 & \text { Conclusion and discussion } & 17\end{array}$

\section{Introduction}

The breakdown of the low-energy effective theory is anticipated [1, 2] for resolving the information loss paradox [3]. But how does the effective theory break down? Assuming string theory, it is argued that the effective theory breaks down due to longitudinal stringstretching effects [4]. On the other hand, there should be clear signals for the breakdown of an effective theory even when we do not know anything about the UV theory.

In this work, we examine the validity of the low-energy effective theory for black-hole physics by carrying out standard QFT calculations under the assumption that the horizon is initially uneventful, as in the conventional model of black holes. Without proposing new physical principles, the only novelty is that we take into consideration the effect of higherderivative interactions, which are ubiquitous in generic low-energy effective theories [5].

Non-renormalizable contributions to a physical quantity (e.g. a scattering amplitude) are typically suppressed by powers of $E^{2} / M_{p}^{2}$, where $E$ is the characteristic energy of the physical process and the Planck mass $M_{p}$ is by assumption the cutoff energy of the effective theory. Hence, non-renormablizable interactions have been ignored in most studies of black-hole physics (except that its potential importance was mentioned in ref. [6]). But they also play the role of an indicator of the validity of the effective theory. The effective theory breaks down whenever non-renormalizable contributions are large, because it means $E \gtrsim M_{p}$, and a UV theory is needed.

In particular, higher-derivative (non-renormalizable) interactions play an important role in black-hole physics for the following reasons. First, due to the trans-Planckian problem $[7,8]$ of Hawking radiation [9-11], ultra-energetic quantum modes must be included in the effective theory. The energy scale $E$ for a process involving these modes can be 
large. Secondly, while the equivalence principle is what prevents the horizon from becoming eventful, higher-derivative interactions have the potential to violate the equivalence principle [12].

In this paper, we will consider a spherical thin null shell in gravitational collapse and compute in this background geometry the transition amplitudes for the following two physical processes: (1) the creation of outgoing particles from the Unruh vacuum due to higher-derivative couplings to the background curvature, ${ }^{1}$ and (2) the decay of an ingoing particle into an ingoing one and two outgoing ones through 4-point higher-derivative interactions. The outgoing particles in both amplitudes are defined from the viewpoint of distant observers, as we use asymptotic states in the calculation of scattering amplitudes. In particular, we consider outgoing particles with energies $\sim \mathcal{O}(1 / a)$ because states including them must exist in order for the effective theory to predict Hawking radiation. (Here, $a \equiv 2 G_{N} M$ is the Schwarzschild radius, where $G_{N}$ is the Newton constant and $M$ is the black hole mass.)

It turns out that these amplitudes grow exponentially with time and become $\mathcal{O}(1)$ at the scrambling time ${ }^{2}$ after the collapsing matter reaches $r-a \sim \mathcal{O}(a)$. At this time, the areal radius of the matter is close to $r=a+\mathcal{O}\left(\ell_{p}^{2} / a\right)$, where $\ell_{p} \equiv 1 / M_{p}$ is the Planck length, and the proper distance between the matter and the horizon is of $\mathcal{O}\left(\ell_{p}\right)$. As a result, the effective theory becomes invalid for physics at the energy scale of Hawking radiation.

The key point behind the particle creations from vacuum is the uncertainty principle for the non-commutativity of the two momentum operators defined with respect to distant observers and freely falling observers. Because of this uncertainty principle, momentum conservation for freely falling observers hardly restricts the creation of outgoing particles for distant observers.

For essentially the same reason, the amplitude for a freely falling particle to radiate outgoing particles to distant observers due to higher-derivative interactions also grows exponentially with time. It may provide a mechanism to transfer information from the infalling particle to outgoing radiation.

The result that the effective theory breaks down around the scrambling time for the energy scale of Hawking radiation means that a UV theory is needed to decide whether Hawking radiation continues and whether the horizon remains uneventful. Regardless of what happens in the UV theory, however, the information loss paradox is no longer a paradox for the effective theory.

The plan of this paper is as follows. We review in section 2 necessary elements about quantum scalar fields in the spacetime background of a matter shell in gravitational collapse. The trans-Planckian problem is reviewed in section 3, and the issue of energy conservation in a local freely falling frame and the uncertainty principle are explained in section 4. The amplitude for the creation of particles due to higher-derivative couplings to the background Ricci tensor is calculated in section 5. In section 6 , we compute the amplitude for the radiation of freely falling particles due to higher-derivative interactions.

\footnotetext{
${ }^{1}$ This particle creation is independent of Hawking radiation, which appears even for free field theories.

${ }^{2}$ The scrambling time $\Delta t \sim \mathcal{O}\left(a \log \left(a M_{p}\right)\right)$ was initially introduced in a different context [13].
} 
Finally, we make comments in section 7 on the generalization of the calculations in this paper and different scenarios for black-hole evaporation.

\section{Review of scalar field in Schwarzschild background}

We review in this section the quantum physics for a black hole with spherical symmetry. ${ }^{3}$ The Schwarzschild metric outside the collapsing matter is

$$
d s^{2}=-\left(1-\frac{a}{r}\right) d u d v+r^{2}(u, v) d \Omega^{2} .
$$

The areal radius $r(u, v)$ is implicitly defined by the equation

$$
\frac{v-u}{2}=r+a \log \left(\frac{r}{a}-1\right)
$$

The light-cone coordinates $(u, v)$ coincide with the retarded and advanced light-cone coordinates in the asymptotically flat region. For simplicity, we consider the gravitational collapse of a null thin shell at $v=v_{s}$, so the metric (2.1) holds only for $v \geq v_{s}$.

In the near-horizon region where $0<r-a \ll a$, the Schwarzschild metric is approximately

$$
d s^{2} \simeq-d U d V+r^{2} d \Omega^{2}
$$

where

$$
\begin{aligned}
& U(u) \simeq-2 a e^{-\frac{u}{2 a}} \\
& V(v) \simeq 2 a e^{\frac{v}{2 a}}
\end{aligned}
$$

are (up to a constant factor) the Kruskal coordinates. These are the coordinates suitable for freely falling observers comoving with the collapsing matter. The red-shift factor between the retarded light-cone coordinates $u$ and $U$,

$$
\frac{d U}{d u} \simeq e^{-\frac{u}{2 a}}
$$

decays exponentially with $u$. The future horizon is located at $u=\infty$ (or equivalently, $U=0)$.

The metric inside the collapsing shell is the Minkowski metric:

$$
d s^{2}=-d U d V+R^{2}(U, V) d \Omega^{2},
$$

where

$$
R(U, V) \equiv \frac{V-U}{2} .
$$

We have used the same symbols $(U, V)$ for these coordinates because they can be identified with the Kruskal coordinates (2.4) and (2.5) across the collapsing shell in the near-horizon region.

\footnotetext{
${ }^{3}$ For a more comprehensive review, see ref. [14].
} 
Ignoring the back-reaction of the vacuum energy-momentum tensor, the energymomentum tensor of the null shell has the only non-vanishing component

$$
T_{V V}=\frac{M}{4 \pi R^{2}(U, V)} \delta\left(V-V_{s}\right),
$$

where $V_{s}=2 a \exp \left(v_{s} / 2 a\right)$ is the $V$-coordinate of the null thin shell. At the horizon, $R\left(0, V_{s}\right)=a$, so eq. (2.8) implies that, in our convention, $v_{s}=0$ and

$$
V_{s}=2 a .
$$

For a very large black hole, the curvature is small. For the spacetime region and the frequencies to be considered below, the $s$-wave of a quantum field $\phi$ is well approximated by

$$
\begin{aligned}
\phi & \simeq \int_{0}^{\infty} \frac{d \Omega}{4 \pi \sqrt{\Omega} R(U, V)}\left(a_{\Omega} e^{-i \Omega U}+a_{\Omega}^{\dagger} e^{i \Omega U}+\tilde{a}_{\Omega} e^{-i \Omega V}+\tilde{a}_{\Omega}^{\dagger} e^{i \Omega V}\right) \\
& =\int_{0}^{\infty} \frac{d \omega}{4 \pi \sqrt{\omega} R(U(u), V(v))}\left(b_{\omega} e^{-i \omega u}+b_{\omega}^{\dagger} e^{i \omega u}+\tilde{b}_{\omega} e^{-i \omega v}+\tilde{b}_{\omega}^{\dagger} e^{i \omega v}\right),
\end{aligned}
$$

where the creation-annihilation operators satisfy the canonical commutation relations:

$$
\begin{aligned}
{\left[a_{\Omega}, a_{\Omega^{\prime}}^{\dagger}\right] } & =\delta\left(\Omega-\Omega^{\prime}\right), & {\left[\tilde{a}_{\Omega}, \tilde{a}_{\Omega^{\prime}}^{\dagger}\right] } & =\delta\left(\Omega-\Omega^{\prime}\right), \\
{\left[b_{\omega}, b_{\omega^{\prime}}^{\dagger}\right] } & =\delta\left(\omega-\omega^{\prime}\right), & {\left[\tilde{b}_{\omega}, \tilde{b}_{\omega^{\prime}}^{\dagger}\right] } & =\delta\left(\omega-\omega^{\prime}\right) .
\end{aligned}
$$

The Unruh vacuum $|0\rangle$ can be defined by

$$
a_{\Omega}|0\rangle=\tilde{a}_{\Omega}|0\rangle=0 .
$$

It can be identified with the Minkowski vacuum of the infinite past.

The creation-annihilation operators $\left(a_{\Omega}^{\dagger}, a_{\Omega}\right)$ are defined with respect to the Kruskal coordinate $U$, and $\left(b_{\omega}^{\dagger}, b_{\omega}\right)$ those defined with respect to fiducial observers (or distant observers). They are related to each other via the Bogoliubov transformation

$$
\begin{aligned}
& b_{\omega}=\int_{0}^{\infty} d \Omega\left(\alpha_{\omega \Omega} a_{\Omega}+\beta_{\omega \Omega} a_{\Omega}^{\dagger}\right), \\
& b_{\omega}^{\dagger}=\int_{0}^{\infty} d \Omega\left(\beta_{\omega \Omega}^{*} a_{\Omega}+\alpha_{\omega \Omega}^{*} a_{\Omega}^{\dagger}\right),
\end{aligned}
$$

where

$$
\begin{aligned}
\alpha_{\omega \Omega} & \equiv \frac{1}{2 \pi} \sqrt{\frac{\omega}{\Omega}} \int_{-\infty}^{\infty} d u e^{i \omega u-i \Omega U(u)} \simeq \frac{a}{\pi} \sqrt{\frac{\omega}{\Omega}}(2 a \Omega)^{i 2 a \omega} e^{\pi a \omega} \Gamma(-i 2 a \omega), \\
\beta_{\omega \Omega} & \equiv \frac{1}{2 \pi} \sqrt{\frac{\omega}{\Omega}} \int_{-\infty}^{\infty} d u e^{i \omega u+i \Omega U(u)} \simeq \frac{a}{\pi} \sqrt{\frac{\omega}{\Omega}}(2 a \Omega)^{i 2 a \omega} e^{-\pi a \omega} \Gamma(-i 2 a \omega),
\end{aligned}
$$

with $U(u)$ given by eq. (2.4) in the near-horizon region.

A proper description of Hawking radiation requires the use of wave packets, as Hawking noticed in his original paper [10], so that one can talk about the radiation at different times during the gravitational collapse. Furthermore, a physical particle is always detected within a finite region of space and time, as opposed to being a uniform spacetime-filling plane wave. 
A wave packet allows us to talk about a particle of an approximate energy $\omega_{0}$ localized within a neighborhood of size $\Delta u$ centered at a certain point $u_{0}$. The uncertainty $\Delta \omega$ in the frequency $\omega_{0}$ and the size $\Delta u$ obey the uncertainty relation $\Delta u \Delta \omega \geq 1$.

For a well-localized wave packet around $u_{0}$ with a given frequency distribution $f_{\omega_{0}}(\omega)$, the corresponding creation operator is ${ }^{4}$

$$
b_{\left(\omega_{0}, u_{0}\right)}^{\dagger}=\int_{0}^{\infty} d \omega f_{\omega_{0}}(\omega) e^{i \omega u_{0}} b_{\omega}^{\dagger},
$$

where $f_{\omega_{0}}(\omega)$ is assumed to be normalized:

$$
\int_{0}^{\infty} d \omega f_{\omega_{0}}^{*}(\omega) f_{\omega_{0}}(\omega)=1
$$

so that $\left[b_{\left(\omega_{0}, u_{0}\right)}, b_{\left(\omega_{0}, u_{0}\right)}^{\dagger}\right]=1$.

As a simple example, we will use the Gaussian wave packet with the frequency distribution

$$
f_{\omega_{0}}(\omega)=c_{\omega_{0}} e^{-\frac{\left(\omega-\omega_{0}\right)^{2}}{2(\Delta \omega)^{2}}}
$$

where $c_{\omega_{0}}$ is determined by the normalization condition (2.20) (up to a phase factor):

$$
c_{\omega_{0}}=\left[\int_{0}^{\infty} d \omega e^{-\frac{\left(\omega-\omega_{0}\right)^{2}}{(\Delta \omega)^{2}}}\right]^{-1 / 2} \simeq\left[\frac{\sqrt{\pi}(\Delta \omega)}{2}\right]^{-1 / 2} \quad \text { for } \omega_{0} \gg(\Delta \omega) .
$$

Hawking radiation can be described in terms of $\left\langle 0\left|b_{\left(\omega_{0}, u_{0}\right)}^{\dagger} b_{\left(\omega_{0}, u_{0}\right)}\right| 0\right\rangle$ for a given wave packet, which is proportional to the Planck distribution [10].

\section{Trans-Planckian problem}

Naively, ${ }^{5}$ the frequency $\omega$ defined with respect to $u$ corresponds to the frequency $\Omega$ defined with respect to $U$ through the red-shift factor $d U / d u$ as

$$
\Omega \sim\left(\frac{d U}{d u}\right)^{-1} \omega
$$

Since Hawking radiation is dominated by the frequencies $\omega \sim \mathcal{O}(1 / a)$, if the low-energy effective theory is only valid for frequencies $\omega$ below a certain cutoff $\Lambda_{\omega}$, we must have

$$
\Lambda_{\omega}>\frac{1}{a}
$$

for Hawking radiation to be a reliable prediction. Through eq. (3.1), one expects that the cutoff $\Lambda_{\omega}$ on $\omega$ is translated to a cutoff $\Lambda_{\Omega}$ on $\Omega$ with

$$
\Lambda_{\Omega}>\left(\frac{d U}{d u}\right)^{-1} \frac{1}{a}
$$

\footnotetext{
${ }^{4}$ Hawking considered wave packets with the frequency distribution $f_{\omega_{0}}(\omega)=\frac{1}{\sqrt{\epsilon}}\left(\Theta\left(\omega-\omega_{0}\right)-\Theta\left(\omega-\omega_{0}-\epsilon\right)\right)$ in his original work [10].

${ }^{5}$ Strictly speaking, as $d U / d u(2.6)$ changes exponentially with time, a constant frequency $\omega$ is mapped to a wide range of frequencies $\Omega$, instead of a unique frequency (3.1). A more precise description of this mapping between $\omega$ and $\Omega$ in terms of wave packets will be given in section 4 .
} 
Over the evaporation of a time scale $\sim \mathcal{O}\left(a^{3} / \ell_{p}^{2}\right)$, say, for Hawking radiation to persist till the Page time, eqs. (2.6) and (3.3) lead to

$$
\Lambda_{\Omega}>\mathcal{O}\left(\frac{1}{a} e^{a^{2} / \ell_{p}^{2}}\right)
$$

Thus, Hawking radiation demands the validity of the effective theory for extremely high frequencies $\Omega$. This is the trans-Planckian problem [7, 8] of Hawking radiation.

Some have argued that this trans-Planckian problem can be dismissed. First of all, it was shown that Hawking radiation persists $[15,16]$ in free-field theories with modified dispersion relations for which the energy is always smaller than the Planck mass $M_{p}{ }^{6}$ Renormalizable interactions were also studied [18-20] and no significant effect on Hawking radiation was found.

A natural next step is to study the effect of higher-derivative non-renormalizable interactions on Hawking radiation, as they amplify the contributions of high-frequency modes. Moreover, the effective theory breaks down whenever their contributions are large.

The potential importance of higher-derivative non-renormalizable interactions were suggested in ref. [6], and it was recently found [21, 22] that, from the viewpoint of distant observers, higher-derivative interactions induce large amplitudes for the creation of particles. However, without using the notion of wave packets, the calculation was not rigorous, and there was an misinterpretation of the result in refs. [21, 22]. We will give a proper interpretation in section 4 on the large amplitudes, and a cleaner and more rigorous derivation of a similar result in section 5 .

Another argument against the trans-Planckian problem is that the frequency $\Omega$ for outgoing particles is not a local Lorentz invariant. It can be arbitrarily large or small after a local Lorentz boost: $U \rightarrow \lambda U\left(\lambda \in \mathbb{R}_{+}\right)$, which corresponds to $\Omega \rightarrow \lambda^{-1} \Omega$. Since the cutoff energy of an effective theory should be locally Lorentz invariant, ${ }^{7}$ the cutoff $M_{p}$ of the effective theory does not imply a cutoff like $M_{p}>\Omega$.

This argument can be refuted by constructing an invariant energy $E$ from $\Omega$. As $\Omega$ is the $U$-component of the momentum (to be denoted by $P_{U}$ ) of a quantum mode, we only need the $V$-component of some momentum (to be denoted by $P_{V}$ ) to define an invariant. In addition to the collapsing matter which clearly has a non-zero $P_{V}$, there is also an ingoing negative vacuum energy flux in the near-horizon region $[23,24]$. In either case, we expect the background to have a $P_{V}$-component no less than $P_{V} \sim 1 / a$. Hence, an invariant characteristic energy can then be defined as

$$
E^{2} \equiv P_{U} P_{V} \sim \frac{\Omega}{a}
$$

When we fix the outgoing momentum to be $\omega \sim \mathcal{O}(1 / a)$, we plug the corresponding $\Omega(3.1)$ into eq. (3.5) and find the invariant energy scale

$$
E^{2} \sim \frac{1}{a^{2}} e^{\frac{u}{2 a}}
$$

\footnotetext{
${ }^{6}$ See ref. [17] for reservations about the implications of these works.

${ }^{7}$ This is why we can apply the standard model to ultra-high-energy cosmic rays.
} 
With the enhancement of higher-derivatives, the amplitude for particle creation, as a function of such an invariant energy $E$, is expected to be of the form

$$
\text { Amplitude } \sim\left(\frac{E^{2}}{M_{p}^{2}}\right)^{m} \sim\left(\frac{\ell_{p}^{2}}{a^{2}} e^{\frac{u}{2 a}}\right)^{m},
$$

where we expect a larger $m$ for a higher-derivative interaction of higher order.

This argument might be considered unreliable because the energy $E$ (3.5) involves the frequency $\Omega$ of a virtual particle, instead of a real one. But we will show explicitly in section 5 that the amplitudes for creating real particles for distant observers due to a class of higher-derivative interactions indeed grow exponentially with time precisely as eq. (3.7) with $m>0$. As a result, at the scrambling time

$$
u \sim 2 a \log \left(a^{2} / \ell_{p}^{2}\right)
$$

the amplitudes become of order 1 , and the effective theory breaks down. We will consider the amplitudes for other processes in section 6 that also grow exponentially with time. The trans-Planckian problem does lead to the breakdown of the effective theory.

An important point to emphasize here is that the created particles with large amplitudes are defined with respect to distant observers, rather than freely falling observers. More precisely, these particles are defined by localized wave packets with approximate frequencies $\omega$. This will be the crucial point in resolving a seemingly paradoxical question about momentum conservation in the freely falling frame in the next section. Another potential concern the reader may have is how a large amplitude (3.7) for non-renormalizable interactions is compatible with the "nice-slice argument" $[25,26]$. We will also answer this question in the next section.

\section{Energy conservation and uncertainty relation}

Naively, the detection of particles of $\omega \sim 1 / a$ on top of Hawking radiation would imply the existence of trans-Planckian particles with extremely large $\Omega$ for freely falling observers. This is obviously in conflict with momentum conservation in the freely falling frame. In this section, we explain why large amplitudes for particle creation at distances are not ruled out by momentum conservation. As we will see below, there are two key points for resolving this conflict. One is to use wave packets to formulate conservation laws in a more precise way. The other is the uncertainty relation for the two momentum operators defined with respect to distant observers and freely falling observers.

First, in the near-horizon region, the translation of $U$ is an approximate symmetry of the metric (2.3). But the translation of $u$ (which is equivalent to a scaling of $U$ without scaling $V$ ) is not a symmetry. ${ }^{8}$ As a result, the component $P_{U}$ of the momentum is approximately conserved but $P_{u}$ is not. Hence, although Hawking radiation carries positive $P_{u}$, it is allowed to emerge from the vacuum.

\footnotetext{
${ }^{8}$ The simultaneous translation of $u$ and $v$ is a symmetry of the Schwarzschild metric, but it is broken by the background of collapsing matter at $v=v_{s}$.
} 
The outgoing particles in Hawking radiation also do not violate $P_{U}$-conservation because Hawking particles are only virtual particles for freely falling observers. On the other hand, the state with 1 outgoing particle on top of Hawking radiation for distant observers,

$$
b_{\omega}^{\dagger}|0\rangle=\int_{0}^{\infty} d \Omega \alpha_{\omega \Omega}^{*} a_{\Omega}^{\dagger}|0\rangle,
$$

is simply a superposition of 1-particle states $a_{\Omega}^{\dagger}|0\rangle$ for freely falling observers, where we have used the Bogoliubov transformation (2.16). Similarly, the state with 2 outgoing particles on top of Hawking radiation for distant observers is a superposition of 2-particle states for freely falling observers because

$$
b_{\omega}^{\dagger} b_{\omega^{\prime}}^{\dagger}|0\rangle=\int_{0}^{\infty} d \Omega \int_{0}^{\infty} d \Omega^{\prime} \alpha_{\omega \Omega}^{*} \alpha_{\omega^{\prime} \Omega^{\prime}}^{*} a_{\Omega}^{\dagger} a_{\Omega^{\prime}}^{\dagger}|0\rangle .
$$

Here we have used the identity

$$
\int_{0}^{\infty} d \Omega \beta_{\omega \Omega}^{*} \alpha_{\omega^{\prime} \Omega}^{*}=0
$$

for $\omega, \omega^{\prime}>0$, which we can check using eqs. (2.17) and (2.18). That is, the particles created on top of Hawking radiation for distant observers correspond to particles on top of the Unruh vacuum for freely falling observers. They carry both positive $P_{u}$ and positive $P_{U}$. Naively, the creation of these particles is expected to violate $P_{U}$-conservation.

Furthermore, even when the outgoing particles have their $P_{u}$ 's as small as $\mathcal{O}(1 / a)$, the corresponding $P_{U}$-values are naively extremely large due to the large blue-shift factor $(d U / d u)^{-1}$ at late times (see eqs. (2.6) and (3.1)). The creation of such high-energy particles should be strongly prohibited by $P_{U}$-conservation. How is it possible to have large amplitudes (like eq. (3.7) for $m>0$ ) for the creation of such particles? We resolve this apparent conceptual problem here.

Let us first review how $P_{U}$-conservation constrains an amplitude. When we calculate scattering amplitudes involving outgoing particles created by $a_{\Omega}^{\dagger}$, which are $P_{U}$-eigenstates, the amplitude is basically an integral over the product of the wave functions (or their complex conjugates) ${ }^{9}$

$$
\left\langle 0\left|\phi(U, V) a_{\Omega}^{\dagger}\right| 0\right\rangle=\frac{1}{4 \pi \sqrt{\Omega} R(U, V)} e^{-i \Omega U}
$$

for each outgoing particle. Apart from additional factors of $\Omega$ for the $U$-derivatives in the interaction term, the amplitude for creating $k$ outgoing particles from the vacuum is proportional to the integral

$$
\int_{-\infty}^{\infty} \frac{d U}{R^{k}(U, V)} e^{-i\left(\Omega_{1}+\cdots+\Omega_{k}\right) U}
$$

If $R(U, V)$ is $U$-independent, the integral is proportional to the Dirac $\delta$-function $\delta\left(\sum_{i=1}^{k} \Omega_{i}\right)$, which imposes $P_{U}$-conservation. For the consideration of particle creation from the vacuum,

\footnotetext{
${ }^{9}$ Please refer to sections 5 and 6 for the explicit calculations.
} 
all particles have positive frequencies $\Omega_{i}>0$, so that $\delta\left(\sum_{i=1}^{k} \Omega_{i}\right)=0$, and the amplitude vanishes. In general, for a smooth function $R(U, V)$ of $U$ with the characteristic length scale $L$, the integral (4.5) for $\sum_{i=1}^{k} \Omega_{i} \gg 1 / L$ is very small.

However, $R(U, V)$ can be well-defined in an effective theory only if it is much larger than the Planck length $\ell_{p}$. The integral (4.5) is ill-defined for a gravitational collapse in which $R$ goes to 0 within finite $U$. One may thus wish to compute the amplitude up to a certain moment $U=U_{*}$ (e.g. up to the event horizon at $U=0$ ). The integral (4.5) is then replaced by

$$
\int_{-\infty}^{U_{*}} \frac{d U}{R^{k}(U, V)} e^{-i\left(\Omega_{1}+\cdots+\Omega_{k}\right) U}
$$

which is non-zero even when all $\Omega_{i}$ 's are positive. That is, for any finite $U_{*}, P_{U^{-}}$ conservation can be violated. The standard interpretation in quantum mechanics is that the measurement at an exact moment $U=U_{*}$ introduces an uncertainty in the momentum $P_{U}$ through the uncertainty relation $\Delta U \Delta P_{U} \gtrsim 1$.

In a low-energy effective theory, it is unphysical to introduce an instant $U_{*}$ with perfect precision. We can avoid this problem using the notion of wave packets. Consider a particle $a_{\left(\Omega_{0}, U_{0}\right)}^{\dagger}|0\rangle$, where

$$
a_{\left(\Omega_{0}, U_{0}\right)}^{\dagger} \equiv \int_{0}^{\infty} d \Omega f_{\Omega_{0}}(\Omega) e^{i \Omega_{0} U_{0}} a_{\Omega}^{\dagger}
$$

with the Gaussian wave packet (2.21) (with $\Delta \omega$ replaced by $\Delta \Omega$ ). Its wave function is

$$
\left\langle 0\left|\phi(U, V) a_{\left(\Omega_{0}, U_{0}\right)}^{\dagger}\right| 0\right\rangle \sim \frac{(\Delta \Omega)^{1 / 2}}{4 \pi^{3 / 4} \Omega_{0}^{1 / 2} R(U, V)} e^{-\frac{(\Delta \Omega)^{2}\left(U-U_{0}\right)^{2}}{2}} e^{-i \Omega_{0}\left(U-U_{0}\right)} .
$$

(We shall not assume that $\Omega_{0} \gg \Delta \Omega$ for the discussion below. But even when $\Omega_{0} \ll \Delta \Omega$, there is only a factor of 2 difference in the approximation above.)

The amplitude for the creation of, say, two particles in the state $a_{\left(\Omega_{1}, U_{1}\right)}^{\dagger} a_{\left(\Omega_{2}, U_{2}\right)}^{\dagger}|0\rangle$ would then be independent of the choice of $U_{*}$ as long as their wave packets reside well within the range $\left(-\infty, U_{*}\right)$. One may then replace the range $\left(-\infty, U_{*}\right)$ by $(-\infty, \infty)$ in the integral of the wave functions. For example, for $k=2$, the amplitude (4.6) is replaced by

$$
\begin{aligned}
\int_{-\infty}^{\infty} & d U\left\langle 0\left|\phi(U, V) a_{\left(\Omega_{1}, U_{1}\right)}^{\dagger}\right| 0\right\rangle\left\langle 0\left|\phi(U, V) a_{\left(\Omega_{2}, U_{2}\right)}^{\dagger}\right| 0\right\rangle \\
& \simeq \frac{e^{i \frac{\left(\Omega_{2}-\Omega_{1}\right)\left(U_{2}-U_{1}\right)}{2}}}{16 \pi \sqrt{\Omega_{1} \Omega_{2}} R^{2}\left(\left(U_{1}+U_{2}\right) / 2, V\right)} e^{-\frac{(\Delta \Omega)^{2}\left(U_{2}-U_{1}\right)^{2}}{4}} e^{-\frac{\left(\Omega_{1}+\Omega_{2}\right)^{2}}{4(\Delta \Omega)^{2}}}
\end{aligned}
$$

where we have assumed that $R(U, V)$ is approximately a constant in a small neighborhood of the width $\Delta U$ around $U=\left(U_{1}+U_{2}\right) / 2$. This is a valid assumption as long as $\Delta U \ll a$.

The exponential factor $\exp \left(-(\Delta \Omega)^{2}\left(U_{2}-U_{1}\right)^{2} / 4\right)$ in eq. (4.9) reflects the locality of the interaction. The last exponential factor $\exp \left(-\left(\Omega_{1}+\Omega_{2}\right)^{2} / 4(\Delta \Omega)^{2}\right)$ imposes the requirement of the approximate $P_{U}$-conservation: $\Omega_{1}+\Omega_{2} \simeq 0$. The amplitude is exponentially suppressed when $P_{U}$-conservation is significantly violated, i.e. when $\left(\Omega_{1}+\Omega_{2}\right) / \Delta \Omega \gg$ 1. As we can choose wave packets with small $\Delta \Omega$, we see that only the states with low frequencies $(\Omega \sim 0)$ have a significant probability to be created from the vacuum. This is a more precise formulation of $P_{U}$-conservation in quantum theory. 
However, the situation is quite different from the perspective of distant observers. In quantum mechanics, we define the momentum operators

$$
P_{u} \equiv-i \hbar \partial_{u}, \quad P_{U} \equiv-i \hbar \partial_{U} \quad \Rightarrow \quad P_{u}=\frac{d U}{d u} P_{U}=e^{-\frac{u}{2 a}} P_{U}
$$

where we have used eq. (2.6). The operator $P_{u}$ does not commute with $P_{U}$. Instead,

$$
\left[P_{u}, P_{U}\right]=\frac{i \hbar}{2 a} P_{U}
$$

which implies the uncertainty relation:

$$
(\Delta \omega)(\Delta \Omega) \gtrsim \frac{\Omega}{a},
$$

where $\Omega(\omega)$ is the expectation value of $P_{U}\left(P_{u}\right)$ for a given state, and $\Delta \Omega(\Delta \omega)$ the standard deviation.

From the viewpoint of a distant observer, the validity of the effective theory on its prediction of Hawking radiation demands the existence of the states with wave packets satisfying $\Delta \omega \lesssim \omega \sim 1 / a$. For such wave-packet states, we have

$$
\Delta \Omega \gtrsim \Omega
$$

as a result of eq. (4.12). From eq. (4.9), we have seen that $P_{U}$-conservation only forbids the creation of particles with $\Omega_{1}+\Omega_{2}+\cdots \gg \Delta \Omega$. Because of eq. (4.13), we conclude that $P_{U}$-conservation has little constraint on outgoing particles with such wave packets $\omega \sim \mathcal{O}(1 / a) \gtrsim \Delta \omega$. This explains how the detection of particles at distances is compatible with momentum conservation in the freely falling frame.

Note that the detection of such particles for distant observers does not imply the detection of high-frequency particles (those with large $\Omega$ and $\Omega \gg \Delta \Omega$ ) for freely falling observers ${ }^{10}$ even though the former is simply a superposition of the latter (like eq. (4.2)). That is, the $S$-matrix is totally different for different wave packets - it depends on whether they are suitable for the coordinate $u$ or $U$. As the creation of high-frequency particles from the vacuum is prohibited by $P_{U}$-conservation, the interpretation of the large amplitude of particle creation for distant observers as a signal of firewall at the horizon in refs. [21, 22] is not justified (at least in the analysis so far).

We are now ready to comment on the nice-slice argument $[25,26]$. It states that the Hamiltonian evolution of nice time slices should not create excitations of energies $\gg \mathcal{O}(1 / a)$ according to the adiabatic theorem. Naively, it implies that no UV theory is needed here.

As far as we have checked, the horizon remains uneventful for freely falling observers as we explained below eq. (4.9), so the effective theory continues to be valid to describe low-energy physics around the horizon. Furthermore, the outgoing particles are of small energies for distant observers. The nice-slice argument is valid in the sense that there is no high-energy (real) event on nice slices.

\footnotetext{
${ }^{10}$ This is because a particle with large $\Omega$ but $\Delta \Omega>\Omega$ can have a frequency anywhere in the range $(\Omega-\Delta \Omega, \Omega+\Delta \Omega)$, which can be very small.
} 
On the other hand, the large energy scale $E$ (3.6) responsible for large amplitudes for higher-derivative interactions is locally Lorentz-invariant, completely independent of the choice of time slices. If the time slices are chosen such that the momentum $P_{V}$ of the infalling matter is small, the momentum $P_{U}$ of an outgoing virtual Hawking particle must be large. (That is the case for the conventional construction of nice slices [25].) Although this trans-Planckian interaction does not lead to high-energy events because it is associated with virtual particles, its proper description still demands a UV theory.

In short, the nice-slice argument may exclude high-energy (real) events, but we still need a UV theory to describe processes involving virtual particles with an invariant transPlanckian energy. In the following, we shall identify such physical processes, and show how they are in need of a UV description.

\section{Particle creation by higher-derivative interactions}

In this section, as an explicit demonstration of the general arguments in sections 3 and 4 , we compute the amplitude of particle creation due to higher-derivative couplings to the background curvature. As examples, we consider

$$
\hat{\mathcal{O}}_{n} \equiv \frac{g_{n}}{2} g^{\mu_{1} \lambda_{1}} \cdots g^{\mu_{n} \lambda_{n}} g^{\nu_{1} \rho_{1}} \cdots g^{\nu_{n} \rho_{n}} R_{\mu_{1} \nu_{1}} \cdots R_{\mu_{n} \nu_{n}}\left(\nabla_{\lambda_{1}} \cdots \nabla_{\lambda_{n}} \phi\right)\left(\nabla_{\rho_{1}} \cdots \nabla_{\rho_{n}} \phi\right),
$$

where $g_{n}$ is the coupling constant and $R_{\mu \nu}$ the Ricci tensor. The dimension of $g_{n}$ is $-(4 n-2)$, so we expect

$$
g_{n} \sim \mathcal{O}\left(1 / M_{p}^{4 n-2}\right) .
$$

The background due to the collapsing null shell is described in section 2. The Ricci tensor is determined by the Einstein equation through eq. (2.9):

$$
R_{V V}(U, V)=\frac{a}{R^{2}} \delta_{d}\left(V-V_{s}\right) .
$$

Here, $\delta_{d}(V)$ denotes a regularized $\delta$-function. It has a support of the size $d$ and a peak of the height $\sim 1 / d$. We shall assume that the thickness of the shell $d \ll a$, and that the characteristic length scales of functions $f(V)$ to be considered below are much larger than $d$, so that

$$
\delta_{d}\left(V-V_{s}\right) f(V) \simeq \delta_{d}\left(V-V_{s}\right) f\left(V_{s}\right) .
$$

We also have

$$
\delta_{d}\left(V-V_{s}\right) \delta_{d}\left(V-V_{s}\right) \simeq \frac{1}{d} \delta_{d}\left(V-V_{s}\right) .
$$

The interaction (5.1) can now be approximated by

$$
\hat{\mathcal{O}}_{n}(U, V) \simeq \frac{-g_{n}(-2)^{n-1} a^{n}}{d^{n-1} R^{2 n}\left(U, V_{s}\right)} \delta_{d}\left(V-V_{s}\right)\left[\nabla_{U}^{n} \phi\left(U, V_{s}\right)\right]^{2} .
$$

The goal of this section is to compute the amplitude for the pair creation of particles from the Unruh vacuum for the interaction (5.1):

$$
\mathcal{M}_{n} \equiv \int d^{4} x \sqrt{-g}\left\langle f\left|\hat{\mathcal{O}}_{n}\right| i\right\rangle
$$


The initial state $|i\rangle$ is the Unruh vacuum $|0\rangle$, and the final state

$$
|f\rangle \equiv b_{\left(\omega_{1}, u_{1}\right)}^{\dagger} b_{\left(\omega_{2}, u_{2}\right)}^{\dagger}|0\rangle
$$

is a state with 2 extra particles on top of Hawking radiation for distant observers, which is equivalently a superposition of 2-particle states in a local freely falling frame (see eq. (4.2)).

To compute the amplitude (5.7), we need to evaluate

$$
\left\langle 0\left|\left(\nabla_{U}^{n} \phi\right)^{2} b_{\left(\omega_{1}, u_{1}\right)}^{\dagger} b_{\left(\omega_{2}, u_{2}\right)}^{\dagger}\right| 0\right\rangle .
$$

Using eqs. (2.19), (4.2) and $a_{\Omega}|0\rangle=0$, it reduces to the product of two factors each of the form $\left\langle 0\left|\nabla_{U}^{n} \phi(U, V) b_{\left(\omega_{0}, u_{0}\right)}^{\dagger}\right| 0\right\rangle$.

To estimate $\left\langle 0\left|\nabla_{U}^{n} \phi(U, V) b_{\left(\omega_{0}, u_{0}\right)}^{\dagger}\right| 0\right\rangle$, we assume that

$$
\frac{1}{R\left(U, V_{s}\right)} \ll \Omega
$$

so that the covariant derivative $\nabla_{U}$ can be approximated by the partial derivative $\partial_{U}$. Using eqs. (2.4), (2.11), (2.12), (2.17), (2.19), as well as the identity

$$
\int_{0}^{\infty} \frac{d \Omega}{\sqrt{\Omega}}(i \Omega)^{n} \alpha_{\omega \Omega} e^{i \Omega U}=\frac{A_{n}(\omega)}{(2 a)^{n}} \frac{e^{i \omega u}}{\sqrt{\omega}} \frac{1}{1-e^{-4 \pi a \omega}} e^{\frac{n u}{2 a}} \quad(n \geq 1),
$$

where

$$
A_{n}(\omega) \equiv(n-1+2 i a \omega)(n-2+2 i a \omega) \cdots(2 i a \omega),
$$

we find

$$
\begin{aligned}
\left\langle 0\left|\nabla_{U}^{n} \phi(u, v) b_{\left(\omega_{0}, u_{0}\right)}^{\dagger}\right| 0\right\rangle & \simeq \int_{0}^{\infty} d \omega f_{\omega_{0}}(\omega) \frac{A_{n}^{*}(\omega)}{(2 a)^{n}} \frac{e^{-i \omega\left(u-u_{0}\right)}}{4 \pi \sqrt{\omega} R} \frac{1}{1-e^{-4 \pi a \omega}} e^{\frac{n u}{2 a}} \\
& =\frac{1}{4 \pi R} \frac{1}{(2 a)^{n}} F_{\left(n, \omega_{0}\right)}^{*}\left(u-u_{0}\right) e^{\frac{n u}{2 a}}
\end{aligned}
$$

where

$$
F_{\left(n, \omega_{0}\right)}(u) \equiv \int_{0}^{\infty} \frac{d \omega}{\sqrt{\omega}} \frac{f_{\omega_{0}}^{*}(\omega) A_{n}(\omega)}{1-e^{-4 \pi a \omega}} e^{i \omega u}
$$

A physical particle should be described by a wave packet with a finite span in spacetime. For simplicity, we consider a Gaussian wave packet (2.21), for which $F_{\left(n, \omega_{0}\right)}(u)(5.15)$ is approximately a Gaussian

$$
F_{\left(n, \omega_{0}\right)}(u) \simeq c_{\omega_{0}} \frac{\sqrt{2 \pi}(\Delta \omega)}{2 \sqrt{\omega_{0}}} \frac{A_{n}\left(\omega_{0}\right)}{1-e^{-4 \pi a \omega_{0}}} e^{i \omega_{0} u} e^{-\frac{(\Delta \omega)^{2} u^{2}}{2}}
$$

assuming that $\omega_{0} \gg \Delta \omega .^{11}$

We shall express the amplitude (5.7) in terms of the central coordinate

$$
u_{c} \equiv \frac{u_{1}+u_{2}}{2},
$$

\footnotetext{
${ }^{11}$ More precisely, the condition is $\frac{\omega_{0}}{\Delta \omega} \gtrsim \frac{u}{\Delta u}=u \Delta \omega$.
} 
of the two particles, and the separation

$$
\delta u \equiv u_{2}-u_{1}
$$

between them. We expect that the amplitude is larger when $\delta u \simeq 0$ since the two particles are created from a local interaction, as in eq. (4.9). We want to understand how the amplitude depends on $u_{c}$, e.g., whether the amplitude is larger at a later time.

Using eqs. (5.6), (5.14), and (5.15), we calculate the amplitude (5.7) as

$$
\mathcal{M}_{n} \simeq \frac{g_{n}(-1)^{n}}{4 \pi d^{n-1}(2 a)^{n}} G_{\left(n, \omega_{1}, \omega_{2}\right)}(\delta u) e^{\frac{(2 n-1) u_{c}}{2 a}},
$$

where $^{12}$

$$
G_{\left(n, \omega_{1}, \omega_{2}\right)}(\delta u) \equiv \int_{-\infty}^{\infty} d u \frac{e^{\frac{(2 n-1)\left(u-u_{c}\right)}{2 a}}}{R^{2 n}\left(U(u), V_{s}\right)} F_{\left(n, \omega_{1}\right)}\left(u-u_{c}+\delta u / 2\right) F_{\left(n, \omega_{2}\right)}\left(u-u_{c}-\delta u / 2\right) .
$$

We have used eq. (2.4) to rewrite the integral of $U$ as an integral of $u$. At large $u_{c}$, the radius $R \simeq a$ is approximately a constant, and we can shift the integration variable $u$ to absorb $u_{c}$ in eq. $(5.20)$, so that $G_{\left(n, \omega_{1}, \omega_{2}\right)}(\delta u)$ becomes insensitive to $u_{c}$. Hence, the amplitude (5.19) increases exponentially with $u_{c}$ as

$$
\mathcal{M}_{n} \propto e^{\frac{(2 n-1) u_{c}}{2 a}} .
$$

Notice that the exponential growth with $u_{c}$ in the amplitude (5.21) is independent of the details of the wave packets, and that it is a faster exponential growth for a higherderivative interaction (5.1) with a larger $n .{ }^{13}$ Only the overall factor $G_{\left(n, \omega_{1}, \omega_{2}\right)}(\delta u)$ in eq. (5.19) depends on the shape of wave packets.

Using eq. (5.16), we find

$$
\left|G_{\left(n, \omega_{1}, \omega_{2}\right)}(\delta u)\right| \gtrsim \frac{\pi^{3 / 2}\left|A_{n}\left(\omega_{1}\right) A_{n}\left(\omega_{2}\right)\right|}{\sqrt{\omega_{1} \omega_{2}} a^{2 n}\left(1-e^{-4 \pi a \omega_{1}}\right)\left(1-e^{-4 \pi a \omega_{2}}\right)} e^{\frac{\left(\frac{2 n-1}{2 a}\right)^{2}-\left(\omega_{1}+\omega_{2}\right)^{2}}{4(\Delta \omega)^{2}}} e^{-\frac{(\Delta \omega)^{2}(\delta u)^{2}}{4}}
$$

near $R \simeq a$. The magnitude of the amplitude is then determined by eqs. (5.19) and (5.22) as

$$
\begin{aligned}
\left|\mathcal{M}_{n}\right| \gtrsim & \frac{\sqrt{\pi} g_{n}\left|A_{n}\left(\omega_{1}\right) A_{n}\left(\omega_{2}\right)\right|}{d^{n-1} 2^{n+2} \sqrt{\omega_{1} \omega_{2}} a^{3 n}\left(1-e^{-4 \pi a \omega_{1}}\right)\left(1-e^{-4 \pi a \omega_{2}}\right)} \\
& \times e^{\frac{\left(\frac{2 n-1}{2 a}\right)^{2}-\left(\omega_{1}+\omega_{2}\right)^{2}}{4(\Delta \omega)^{2}}} e^{-\frac{(\Delta \omega)^{2}(\delta u)^{2}}{4}} e^{\frac{(2 n-1) u_{c}}{2 a}} .
\end{aligned}
$$

\footnotetext{
${ }^{12}$ It can be shown that the $u$-integral in $G_{\left(n, \omega_{1}, \omega_{2}\right)}$ (5.20) diverges due to the exponential factor $e^{\frac{(2 n-1)\left(u-u_{c}\right)}{2 a}}$. Physically, any detected wave packet is always well localized, so one is justified to impose an IR cutoff $u_{\infty}$ on the $u$-integral $\int_{-\infty}^{u_{\infty}}$. For the sake of the simplicity of arguments, we shall proceed and reach the same conclusion without worrying about the cutoff.

${ }^{13}$ As the amplitude is an invariant quantity, it is independent of the choice of the coordinate system. In terms of the asymptotic coordinates $(u, v)$, the interaction term (5.1) involves the factor $\left(g^{u v}\right)^{n} \propto$ $(1-a / r)^{-n}$, which blows up at the horizon. In terms of the Kruskal coordinates $(U, V)$, the derivatives $\partial_{U}^{n}$ give a factor of $\Omega^{n}$, where $\Omega(3.1)$ grows exponentially due to the blue-shift factor $(d U / d u)^{-1}$.
} 
Since eq. (5.23) is a result of the low-energy effective theory, it may not be valid for very high-frequencies $\omega_{1}, \omega_{2}$. However, whenever Hawking radiation is considered a valid prediction of the effective theory, states including outgoing particles for distant observers with

$$
\omega_{1}, \omega_{2} \sim \mathcal{O}(1 / a)
$$

must be allowed. Assuming eq. (5.24), the magnitude of the amplitude (5.23) satisfies the bound $^{14}$

$$
\left|\mathcal{M}_{n}\right| \gg\left(\frac{\ell_{p}}{a}\right)^{4 n-2} e^{\frac{\left(\frac{2 n-1}{2 a}\right)^{2}-\left(\omega_{1}+\omega_{2}\right)^{2}}{4(\Delta \omega)^{2}}} e^{-\frac{(\Delta \omega)^{2}(\delta u)^{2}}{4}} e^{\frac{(2 n-1) u_{c}}{2 a}},
$$

where we have used $d \ll a$

The factor $\exp \left\{\left[\left(\frac{2 n-1}{2 a}\right)^{2}-\left(\omega_{1}+\omega_{2}\right)^{2}\right] / 4(\Delta \omega)^{2}\right\}$ in eq. (5.25) is composed of two parts. The first part $\exp \left[\left(\frac{2 n-1}{2 a}\right)^{2} / 4(\Delta \omega)^{2}\right]$ is an enhancement due to the higher derivatives in the interaction $\hat{\mathcal{O}}_{n}(5.1)$. The second part $\exp \left[-\left(\omega_{1}+\omega_{2}\right)^{2} / 4(\Delta \omega)^{2}\right]$ is the suppression due to the non-conservation of the $P_{u}$-momentum. The joint effect can be of $\mathcal{O}(1)$ or larger by choosing $n$ to be sufficiently large. For instance, if $\omega_{1}=\omega_{2}=1 / a$, this factor is larger than 1 as long as $n \geq 3$.

The next factor $\exp \left(-(\Delta \omega)^{2}(\delta u)^{2} / 4\right)$ in eq. (5.25) is expected as the two particles are created in pairs by a local operator. The last factor $\exp \left[(2 n-1) u_{c} / 2 a\right]$ grows exponential with the central coordinate $u_{c}$ of the created particles. Therefore, the amplitude for the creation of outgoing particles at large distances through higher-derivative couplings to the Ricci tensor grows exponentially with time, as we have seen in eq. (3.7).

The calculation above relies on the Bogoliubov transformation which suffers the transPlanckian problem. Yet, as long as Hawking radiation is still assumed to be a valid prediction of the effective theory, the Bogoliubov transformation has to be valid for the dominant modes of Hawking radiation, i.e. $\omega \sim 1 / a$, and the result (5.25) for the case (5.24) should be valid. For this case, we have (as expected above in eq. (3.7))

$$
\left|\mathcal{M}_{n}\right| \gtrsim\left[\frac{\ell_{p}^{2}}{a^{2}} e^{\frac{u_{c}}{2 a}}\right]^{2 n-1}
$$

for $(2 n-1) \geq 2 a\left(\omega_{1}+\omega_{2}\right)$ and $u_{2} \simeq u_{1}$. When the magnitude of the transition amplitude is $\sim \mathcal{O}(1)$, the low-energy effective theory breaks down. This happens when

$$
u_{c} \gtrsim u_{B} \equiv 2 a \log \left(a^{2} / \ell_{p}^{2}\right),
$$

which is of the same order as the scrambling time [13]. According to eqs. (2.4), (2.8), and (2.10), the $u$-coordinate is defined such that the collapsing shell has the radius $R \simeq 2 a$ at $u=0$. Thus, its radius satisfies

$$
R\left(u_{B}, v_{s}\right)-a \simeq \ell_{p}^{2} / a
$$

at the scrambling time.

\footnotetext{
${ }^{14}$ If eq. (5.24) is replaced by $\omega_{1}, \omega_{2} \gg \mathcal{O}(1 / a)$, the amplitude is larger by a factor of $\left(a \omega_{1}\right)^{n}\left(a \omega_{2}\right)^{n}$. For the case of $\omega_{1}, \omega_{2} \ll \mathcal{O}(1 / a)$, it is smaller by a factor of $\left(a \omega_{1}\right)\left(a \omega_{2}\right)$. The discussion below will be essentially the same.
} 
We conclude that, by the scrambling time $u_{B}$ after passing through the point $R=2 a$ (equivalently, when the matter shell has $R=a+\mathcal{O}\left(\ell_{p}^{2} / a\right)$ ), the amplitude increases to $\mathcal{O}(1)$, and the effective theory breaks down for the outgoing modes with $\omega \gtrsim \mathcal{O}(1 / a)$. It is thus also the last moment when Hawking radiation is a valid prediction of the effective theory.

\section{$6 \quad$ Infalling particle radiation}

In this section, as another explicit example of the general discussions in sections 3 and 4 , we compute the transition amplitude for the decay of an infalling massless particle via 4-point higher-derivative interactions. It includes the case of an infalling particle radiating two outgoing particles. The outgoing particles are defined with respect to distant observers as in section $5 .^{15}$

Introduce several massless scalar fields $\phi_{A}$ and $\psi_{A}(A=1,2)$, each with the same mode expansion as $\phi$ (2.11). For our discussion below, we will only need the ingoing modes of $\phi_{A}$ and the outgoing modes of $\psi_{A}$, and the corresponding creation-annihilation operators are denoted $\left(\tilde{a}_{A \Omega}, \tilde{a}_{A \Omega}^{\dagger}\right)$ and $\left(b_{A \omega}, b_{A \omega}^{\dagger}\right)$, respectively.

We consider the following higher-derivative interactions among these fields:

$$
\begin{aligned}
\hat{\mathcal{O}}_{m n} \equiv & \lambda_{m n} g^{\mu_{1} \nu_{1}} \cdots g^{\mu_{m} \nu_{m}} g^{\lambda_{1} \rho_{1}} \cdots g^{\lambda_{n} \rho_{n}}:\left(\nabla_{\mu_{1}} \cdots \nabla_{\mu_{m}} \phi_{1}\right)\left(\nabla_{\lambda_{1}} \cdots \nabla_{\lambda_{n}} \phi_{2}\right) \\
& \times\left(\nabla_{\nu_{1}} \cdots \nabla_{\nu_{m}} \psi_{1}\right)\left(\nabla_{\rho_{1}} \cdots \nabla_{\rho_{n}} \psi_{2}\right):
\end{aligned}
$$

where

$$
\lambda_{m n} \sim \mathcal{O}\left(1 / M_{p}^{2(m+n)}\right)
$$

is the coupling constant. With this class of higher-derivative interactions, we now compute the transition amplitude for an ingoing particle $\phi_{1}$ to decay into an ingoing particle $\phi_{2}$ and two outgoing particles $\psi_{1}$ and $\psi_{2}$. The initial and final states are

$$
\begin{aligned}
|i\rangle & =\tilde{a}_{1 \Omega}^{\dagger}|0\rangle, \\
|f\rangle & =b_{1\left(\omega_{1}, u_{1}\right)}^{\dagger} b_{2\left(\omega_{2}, u_{2}\right)}^{\dagger} \tilde{a}_{2 \Omega^{\prime}}^{\dagger}|0\rangle,
\end{aligned}
$$

and we shall calculate the amplitude

$$
\mathcal{M}_{m n} \equiv \int d^{4} x \sqrt{-g}\left\langle f\left|\hat{\mathcal{O}}_{m n}\right| i\right\rangle .
$$

For this process, we can simplify the interaction (6.1) as

$$
\hat{\mathcal{O}}_{m n} \simeq \lambda_{m n}\left(g^{U V}\right)^{m+n}\left(\partial_{V}^{m} \phi_{1}\right)\left(\partial_{V}^{n} \phi_{2}\right)\left(\partial_{U}^{m} \psi_{1}\right)\left(\partial_{U}^{n} \psi_{2}\right),
$$

assuming that eq. (5.10) applies to all the relevant frequencies. Then, we just have to calculate

$$
\begin{aligned}
& \left\langle f\left|\hat{\mathcal{O}}_{m n}\right| i\right\rangle \\
& \simeq \lambda_{m n}\left(g^{U V}\right)^{m+n}\left\langle 0\left|\partial_{V}^{m} \phi_{1} \tilde{a}_{1 \Omega}^{\dagger}\right| 0\right\rangle\left\langle 0\left|\partial_{V}^{n} \phi_{2} \tilde{a}_{2 \Omega^{\prime}}^{\dagger}\right| 0\right\rangle^{*}\left\langle 0\left|\partial_{U}^{m} \psi_{1} b_{1\left(\omega_{1}, u_{1}\right)}^{\dagger}\right| 0\right\rangle^{*}\left\langle 0\left|\partial_{U}^{n} \psi_{2} b_{2\left(\omega_{2}, u_{2}\right)}^{\dagger}\right| 0\right\rangle^{*} .
\end{aligned}
$$

\footnotetext{
${ }^{15}$ We can think of the particle production via couplings to the Ricci tensor in section 5 as an analogue of the radiation by infalling particles in this section in the sense that the radiation through direction interaction in the latter is replaced by an indirect interaction mediated by gravity in the former.
} 
For the ingoing modes, we have

$$
\left\langle 0\left|\partial_{V}^{n} \phi_{A}(U, V) \tilde{a}_{B \Omega}^{\dagger}\right| 0\right\rangle=\delta_{A B} \frac{(-i \Omega)^{n}}{4 \pi \sqrt{\Omega} R} e^{-i \Omega V} .
$$

(For the ingoing modes, it makes no significant difference whether the wave packets are introduced, as long as their frequencies $\Omega$ have small uncertainties $\Delta \Omega \ll \Omega$.) Together, eqs. (5.14) and (6.8) allow us to express the amplitude as

$$
\mathcal{M}_{m n}=i^{m-n} \lambda_{m n} \frac{\Omega^{m+n-1} \delta\left(\Omega-\Omega^{\prime}\right)}{32 \pi^{2} a^{m+n+2}} e^{(m+n-1) \frac{u_{c}}{2 a}} H_{\left(m, n, \omega_{1}, \omega_{2}\right)}(\delta u),
$$

where we have assumed $R \simeq a$ and used eqs. (5.15), (5.17), (5.18), and

$$
H_{\left(m, n, \omega_{1}, \omega_{2}\right)}(\delta u) \equiv \int_{-\infty}^{\infty} d u e^{(m+n-1) \frac{u}{2 a}} F_{\left(m, \omega_{1}\right)}(u+\delta u / 2) F_{\left(n, \omega_{2}\right)}(u-\delta u / 2) .
$$

Similar to $G_{\left(n, \omega_{1}, \omega_{2}\right)}$ (5.22), $H_{\left(m, n, \omega_{1}, \omega_{2}\right)}(\delta u)$ can be evaluated for the Gaussian wave packets (2.21) as

$$
\left|\mathcal{M}_{m n}\right| \sim(a \Omega)^{m+n-1}\left(\frac{\ell_{p}^{2}}{a^{2}}\right)^{m+n} \delta\left(\Omega-\Omega^{\prime}\right) e^{\frac{\left(\frac{m+n-1}{2 a}\right)^{2}-\left(\omega_{1}+\omega_{2}\right)^{2}}{4(\Delta \omega)^{2}}} e^{-\frac{(\Delta \omega)^{2}\left(u_{2}-u_{1}\right)^{2}}{4}} e^{(m+n-1) \frac{u_{c}}{2 a}},
$$

where as in section 5 we have assumed

$$
\omega_{1} \sim \omega_{2} \sim \mathcal{O}(1 / a)
$$

For the particle $\phi_{1}$ to fall into the black hole, its wavelength is restricted to be smaller than $a$, so we have $\Omega \gtrsim 1 / a$, and eq. (6.11) gives a bound on the amplitude as

$$
\left|\mathcal{M}_{m n}\right| \gtrsim\left(\frac{\ell_{p}^{2}}{a^{2}}\right)^{m+n} \delta\left(\Omega-\Omega^{\prime}\right) e^{\frac{\left(\frac{m+n-1}{2 a}\right)^{2}-\left(\omega_{1}+\omega_{2}\right)^{2}}{4(\Delta \omega)^{2}}} e^{-\frac{(\Delta \omega)^{2}\left(u_{2}-u_{1}\right)^{2}}{4}} e^{(m+n-1) \frac{u_{c}}{2 a}} .
$$

The interpretation of the last 3 factors in eq. (6.13) is analogous to those in eq. (5.25). In short, there is an exponentially increasing amplitude for the infalling particle to send a signal to distant observers about its presence in the form of two coincident particles $\psi_{1}$ and $\psi_{2}$, and the amplitude becomes of $\mathcal{O}(1)$ at the scrambling time.

The Dirac $\delta$-function in eq. (6.13) demands that the particle $\phi_{1}$ passes all of its momentum to the particle $\phi_{2}$, as a result of the translation symmetry in $V$. When $\phi_{2}=\phi_{1}$, this is the amplitude for an infalling particle to radiate two outgoing particles $\psi_{1}, \psi_{2}$. The radiation has no back-reaction, analogous to the radiation of a freely falling point charge [27-31].

The higher-derivative interaction thus provides a mechanism to transfer (at least part of) the information of the collapsing matter into out-going particles. The time it takes for the information transfer is roughly speaking the scrambling time. This is reminiscent of the original meaning of the scrambling time [13].

How much information of the ingoing particle can be transferred to large distances? If the theory includes both interactions $\partial_{V}^{m} \phi_{1} \partial_{V}^{n} \phi_{1} \partial_{U}^{m} \psi_{1} \partial_{U}^{n} \psi_{2}$ and $\partial_{V}^{m} \phi_{2} \partial_{V}^{n} \phi_{2} \partial_{U}^{m} \psi_{1} \partial_{U}^{n} \psi_{2}$, when coincident outgoing particles $\psi_{1}$ and $\psi_{2}$ are detected, the infalling particle could be either $\phi_{1}$ or $\phi_{2}$. It is interesting to contemplate the possibility that all information of the infalling particles can be retrieved this way for certain UV theories. 


\section{Conclusion and discussion}

In the above, we have considered two specific types of higher-derivative interactions (5.1) and (6.1). The conclusion about exponentially increasing amplitudes (like eq. (3.7) with $m>0$ ) applies to a much wider class of higher-derivative interactions. For example, we can consider more general higher-derivative interactions built from the following invariant (dimensionless) building blocks:

$$
\begin{aligned}
M_{p}^{4} \int d^{4} x \sqrt{-g}=M_{p}^{4} \int d U d V 4 \pi R^{2} & \rightarrow \frac{a^{4}}{\ell_{p}^{4}} e^{-\frac{u}{2 a}}, \\
\frac{1}{M_{p}^{2}} g^{U V} \partial_{U} \otimes \partial_{V} & \rightarrow e^{\frac{u}{2 a}} \frac{\ell_{p}^{2}}{a^{2}} \\
\frac{1}{M_{p}^{4}} R_{V V} g^{U V} g^{U V} \partial_{U} \otimes \partial_{U} & \rightarrow\left(\frac{\ell_{p}^{2}}{a^{2}} e^{\frac{u}{2 a}}\right)^{2}, \\
\frac{1}{M_{p}} \phi & \rightarrow \frac{\ell_{p}}{a},
\end{aligned}
$$

where we have used $\partial_{u} \sim \partial_{v} \rightarrow 1 / a, R \rightarrow a, d U / d u \rightarrow e^{-u / 2 a}$, and $d V / d v \rightarrow 1$ to estimate their contributions to the amplitudes. We can use the formulas above for a rough orderof-magnitude estimate of the amplitudes.

For a generic combination of these factors, an amplitude is

$$
\lambda \int d^{4} x \sqrt{-g}\left[g^{U V} \partial_{U} \otimes \partial_{V}\right]^{m}\left[R_{V V} g^{U V} g^{U V} \partial_{U} \otimes \partial_{U}\right]^{n}[\phi]^{\otimes q} \sim \frac{\ell_{p}^{q-2}}{a^{q-2}}\left[\frac{\ell_{p}^{2}}{a^{2}} e^{\frac{u}{2 a}}\right]^{m+2 n-1}
$$

where the coupling constant $\lambda \sim \mathcal{O}\left(M_{p}^{4-2 m-4 n-q}\right)$. For any finite $m, n$, and $q$ as long as $m+2 n>1$, it becomes large at the scrambling time.

Note that the onset of the breakdown of the effective theory is robustly at the scrambling time. For instance, by increasing the value of $q$, the critical time when a given interaction becomes $\mathcal{O}(1)$ is postponed by an amount $\sim \mathcal{O}\left(2 a \log \left(a / \ell_{p}\right)\right)$, but it is still the same order of magnitude as the scrambling time.

Another type of generalization is to consider other final states. For instance, if we replace the 1-particles state $b_{\omega}^{\dagger}|0\rangle$ by $b_{\omega}|0\rangle$, the wave function (5.13) is replaced by

$$
\left\langle 0\left|\partial_{U}^{n} \phi b_{\left(\omega_{0}, u_{0}\right)}\right| 0\right\rangle \simeq-\int_{0}^{\infty} d \omega f_{\omega_{0}}(\omega) \frac{A_{n}^{*}(-\omega)}{(2 a)^{n}} \frac{e^{i \omega\left(u-u_{0}\right)}}{4 \pi \sqrt{\omega} R} \frac{e^{-4 \pi a \omega}}{1-e^{-4 \pi a \omega}} e^{\frac{n u}{2 a}} .
$$

The same exponential factor $e^{\frac{n u}{2 a}}$ appears here. It is then straightforward to see that the same higher-derivative interaction would also have an exponentially growing amplitude for "removing" particles (with the operator $b_{\left(\omega_{0}, u_{0}\right)}$ ) from Hawking radiation.

Our conclusion is that the effective theory breaks down for any physical process involving outgoing particles with $\omega \gtrsim 1 / a$ (including Hawking radiation) when the collapsing matter is around a Planck length from the horizon. This is reminiscent of the brick-wall model $[7,32]$ (although the horizon may still remain uneventful for freely falling observers 
in our discussion). It will be interesting to explore further connection between our results and the brick-wall model, including its holographic dual [33-35].

Let us now speculate on what could happen after the effective theory breaks down. A simple possibility is that the UV theory imposes an effective cutoff that stops both Hawking radiation and the large amplitude for particle creation. The black hole becomes eternal, perhaps as a "fuzzball" [36, 37]. It is also possible that an effective theory will be valid after a transition through a UV-mechanism, possibly with the Boulware vacuum at the horizon.

Another possibility is that the UV theory keeps the large (but now regulated) amplitude of particle creation, and a large outgoing energy flux (which can be identified with the "firewall" [2] ${ }^{16}$ ) appears around the horizon.

In addition, when an outgoing energy flux appears around the horizon, the spacetime geometry is modified. The Schwarzschild geometry is no longer applicable. Such a scenario was proposed in refs. [39, 40]. Radiation from the collapsing matter induces a large tangential pressure on the collapsing shell [41]. As alternative approaches, connections between stress energy tensor and geometry via the semi-classical Einstein equation have been explored in refs. [42-46].

It will be interesting to find the relation between different scenarios for the black-hole evaporation and the corresponding features in the UV theory. We leave this problem for future investigation.

To conclude, we have established in this paper that the effective-field-theoretic derivation of Hawking radiation is no longer reliable after the scrambling time. Planckian physics decides what happens next. The original argument for information loss due to Hawking radiation based on effective-theory calculations can be dismissed, so the original paradox is resolved in this sense. On the other hand, it is still unclear by what kind of UV mechanism the information is preserved. The Planckian information problem persists.

\section{Acknowledgments}

We thank Heng-Yu Chen, Jiunn-Wei Chen, Yu-tin Huang, Samir Mathur, Nobuyoshi Ohta, Suguru Okumura, Wei-Hsiang Shao, Hideaki Takabe, and Naoki Watamura for valuable discussions. P.M.H. is supported in part by the Ministry of Science and Technology, R.O.C. (MOST 110-2112-M-002 -016 -MY3) and by National Taiwan University. H.K. thanks Professor Shin-Nan Yang and his family for their kind support through the Chin-Yu chair professorship. H.K. is also partially supported by Japan Society of Promotion of Science (JSPS), Grants-in-Aid for Scientific Research (KAKENHI) Grants No. 20K03970 and 18H03708, by the Ministry of Science and Technology, R.O.C. (MOST 110-2811-M-002500), and by National Taiwan University. Y.Y. is partially supported by Japan Society of Promotion of Science (JSPS), Grants-in-Aid for Scientific Research (KAKENHI) Grants No. 21K13929, 18K13550 and 17H01148. Y.Y. is also partially supported by RIKEN iTHEMS Program.

\footnotetext{
${ }^{16}$ See ref. [38] for a similar prediction from different assumptions.
} 
Open Access. This article is distributed under the terms of the Creative Commons Attribution License (CC-BY 4.0), which permits any use, distribution and reproduction in any medium, provided the original author(s) and source are credited.

\section{References}

[1] S.D. Mathur, The information paradox: a pedagogical introduction, Class. Quant. Grav. 26 (2009) 224001 [arXiv:0909.1038] [INSPIRE].

[2] A. Almheiri, D. Marolf, J. Polchinski and J. Sully, Black holes: complementarity or firewalls?, JHEP 02 (2013) 062 [arXiv:1207.3123] [INSPIRE].

[3] S.W. Hawking, Breakdown of predictability in gravitational collapse, Phys. Rev. D 14 (1976) 2460 [INSPIRE].

[4] M. Dodelson and E. Silverstein, String-theoretic breakdown of effective field theory near black hole horizons, Phys. Rev. D 96 (2017) 066010 [arXiv:1504.05536] [INSPIRE].

[5] S. Weinberg, The quantum theory of fields. Volume 1: foundations, Cambridge University Press, Cambridge, U.K. (1995).

[6] S. Iso, Hawking radiation, gravitational anomaly and conformal symmetry: the origin of universality, Int. J. Mod. Phys. A 23 (2008) 2082 [arXiv:0804.0652] [INSPIRE].

[7] G. 't Hooft, On the quantum structure of a black hole, Nucl. Phys. B 256 (1985) 727 [INSPIRE].

[8] T. Jacobson, Black hole evaporation and ultrashort distances, Phys. Rev. D 44 (1991) 1731 [INSPIRE].

[9] S.W. Hawking, Black hole explosions, Nature 248 (1974) 30 [INSPIRE].

[10] S.W. Hawking, Particle creation by black holes, Commun. Math. Phys. 43 (1975) 199 [Erratum ibid. 46 (1976) 206] [INSPIRE].

[11] S.W. Hawking, Black holes and thermodynamics, Phys. Rev. D 13 (1976) 191 [InSPIRE].

[12] R. Lafrance and R.C. Myers, Gravity's rainbow, Phys. Rev. D 51 (1995) 2584 [hep-th/9411018] [INSPIRE].

[13] Y. Sekino and L. Susskind, Fast scramblers, JHEP 10 (2008) 065 [arXiv:0808.2096] [INSPIRE].

[14] R. Brout, S. Massar, R. Parentani and P. Spindel, A primer for black hole quantum physics, Phys. Rept. 260 (1995) 329 [arXiv:0710.4345] [InSPIRE].

[15] W.G. Unruh, Sonic analog of black holes and the effects of high frequencies on black hole evaporation, Phys. Rev. D 51 (1995) 2827 [gr-qc/9409008] [INSPIRE].

[16] R. Brout, S. Massar, R. Parentani and P. Spindel, Hawking radiation without trans-Planckian frequencies, Phys. Rev. D 52 (1995) 4559 [hep-th/9506121] [INSPIRE].

[17] A.D. Helfer, Do black holes radiate?, Rept. Prog. Phys. 66 (2003) 943 [gr-qc/0304042] [INSPIRE].

[18] D.A. Leahy and W.G. Unruh, Effects of a $\lambda \phi^{4}$ interaction on black hole evaporation in two-dimensions, Phys. Rev. D 28 (1983) 694 [INSPIRE].

[19] A.D. Helfer, Quantum character of black holes, gr-qc/0503053 [INSPIRE]. 
[20] M. Frasca, Hawking radiation and interacting fields, Eur. Phys. J. Plus 132 (2017) 467 [arXiv: 1412.1955] [INSPIRE].

[21] P.-M. Ho and Y. Yokokura, Firewall from effective field theory, Universe 7 (2021) 241 [arXiv: 2004.04956] [INSPIRE].

[22] P.-M. Ho, From uneventful horizon to firewall in D-dimensional effective theory, Int. J. Mod. Phys. A 36 (2021) 2150145 [arXiv: 2005. 03817] [INSPIRE].

[23] P.C.W. Davies, S.A. Fulling and W.G. Unruh, Energy momentum tensor near an evaporating black hole, Phys. Rev. D 13 (1976) 2720 [InSPIRE].

[24] S.M. Christensen and S.A. Fulling, Trace anomalies and the Hawking effect, Phys. Rev. D 15 (1977) 2088 [InSPIRE].

[25] D.A. Lowe, J. Polchinski, L. Susskind, L. Thorlacius and J. Uglum, Black hole complementarity versus locality, Phys. Rev. D 52 (1995) 6997 [hep-th/9506138] [INSPIRE].

[26] J. Polchinski, String theory and black hole complementarity, in STRINGS 95: future perspectives in string theory, (1995), pg. 417 [hep-th/9507094] [INSPIRE].

[27] T. Fulton and F. Rohrlich, Classical radiation from a uniformly accelerated charge, Ann. Phys. 9 (1960) 499.

[28] C.M. DeWitt and B.S. DeWitt, Falling charges, Physics Physique Fizika 1 (1964) 3 [Erratum ibid. 1 (1964) 145] [INSPIRE].

[29] J. Cohn, Hyperbolic motion and radiation, Amer. J. Phys. 46 (1978) 225.

[30] R. Peierls, Surprises in theoretical physics, chapter 8, Princeton University Press, Princeton, NJ, U.S.A. (1979)

[31] P. Pearle Classical electron models, in Electromagnetism: paths to research, D. Teplitz ed., Plenum Press, New York, NY, U.S.A. (1982), pg. 211.

[32] G. 't Hooft, The scattering matrix approach for the quantum black hole: an overview, Int. J. Mod. Phys. A 11 (1996) 4623 [gr-qc/9607022] [INSPIRE].

[33] B.S. Kay and L. Ortíz, Brick walls and AdS/CFT, Gen. Rel. Grav. 46 (2014) 1727 [arXiv:1111.6429] [INSPIRE].

[34] N. Iizuka and S. Terashima, Brick walls for black holes in AdS/CFT, Nucl. Phys. B 895 (2015) 1 [arXiv: 1307.5933] [INSPIRE].

[35] S. Terashima, Simple bulk reconstruction in AdS/CFT correspondence, arXiv:2104.11743 [INSPIRE].

[36] O. Lunin and S.D. Mathur, AdS/CFT duality and the black hole information paradox, Nucl. Phys. B 623 (2002) 342 [hep-th/0109154] [INSPIRE].

[37] O. Lunin and S.D. Mathur, Statistical interpretation of Bekenstein entropy for systems with a stretched horizon, Phys. Rev. Lett. 88 (2002) 211303 [hep-th/0202072] [INSPIRE].

[38] S.L. Braunstein, S. Pirandola and K. Życzkowski, Better late than never: information retrieval from black holes, Phys. Rev. Lett. 110 (2013) 101301 [arXiv:0907.1190] [INSPIRE].

[39] H. Kawai, Y. Matsuo and Y. Yokokura, A self-consistent model of the black hole evaporation, Int. J. Mod. Phys. A 28 (2013) 1350050 [arXiv:1302.4733] [InSPIRE].

[40] H. Kawai and Y. Yokokura, Phenomenological description of the interior of the Schwarzschild black hole, Int. J. Mod. Phys. A 30 (2015) 1550091 [arXiv:1409.5784] [InSPIRE]. 
[41] H. Kawai and Y. Yokokura, Black hole as a quantum field configuration, Universe 6 (2020) 77 [arXiv: 2002.10331] [INSPIRE].

[42] P.-M. Ho and Y. Matsuo, On the near-horizon geometry of an evaporating black hole, JHEP 07 (2018) 047 [arXiv: 1804.04821] [INSPIRE].

[43] D. Terno, Self-consistent description of a spherically-symmetric gravitational collapse, Phys. Rev. D 100 (2019) 124025 [arXiv: 1903.04744] [INSPIRE].

[44] P.-M. Ho and Y. Matsuo, Trapping horizon and negative energy, JHEP 06 (2019) 057 [arXiv: 1905.00898] [INSPIRE].

[45] P.-M. Ho, Y. Matsuo and Y. Yokokura, Distance between collapsing matter and apparent horizon in evaporating black holes, Phys. Rev. D 104 (2021) 064005 [arXiv:1912.12863] [INSPIRE].

[46] S. Murk and D.R. Terno, Physical black holes in semiclassical gravity, in $16^{\text {th }}$ Marcel Grossmann meeting on recent developments in theoretical and experimental general relativity, astrophysics and relativistic field theories, (2021) [arXiv:2110.12761] [INSPIRE]. 